\title{
Proper orthogonal decomposition analysis of vortex shedding behind a rotating circular cylinder
}

\author{
Sharul Sham Dol ${ }^{1, a}$ \\ ${ }^{1}$ Petroleum Engineering Department, Curtin University, Sarawak Campus CDT 250, Miri Sarawak, 98009 Malaysia
}

\begin{abstract}
Turbulence studies were made in the wake of a rotating circular cylinder in a uniform free stream with the objective of describing the patterns of the vortex shedding up to suppression of the periodic vortex street at high velocity ratios, $\lambda$. The results obtained in the present study establish that shedding of Kármán vortices in a rotating circular cylinder-generated wake is modified by rotation of the cylinder. Alternate vortex shedding is highly visible when $\lambda<2.0$ although the strength of the separated shear layers differ due to the rotation of the cylinder. The spectral density in the wakes indicate significant changes at $\lambda=2.0$. The results indicate that the rotation of the cylinder causes significant disruption in the structure of the flow. Alternate vortex shedding is weak, distorted and close to being suppressed at $\lambda=2.0$. It is clear that flow asymmetries will weaken vortex shedding, and when the asymmetries are significant enough, total suppression of a periodic street occurs. Particular attention was paid to the decomposition of the flow using Proper Orthogonal Decomposition (POD). By analyzing this decomposition with the help of Particle Image Velocimetry (PIV ) data, it was found that large scales contribute to the coherent motion. Vorticity structures in the modes become increasingly irregular with downstream distance, suggesting turbulent interactions are occurring at the more downstream locations, especially when the cylinder rotates.
\end{abstract}

\section{Introduction}

The study of vortices (i.e. vortex motions, vortex instability, vortex breakdown) will lead to an understanding of turbulent flow. Turbulence is rotational and characterized by high levels of fluctuating vorticity. For this reason, vortex dynamics plays an essential role in the description of turbulent flows. For example, the existence of energy transfer (energy cascade) from large eddies to small eddies is driven by vortex-stretching mechanism. This is an important concept to explain why turbulent flows are always dissipative.

The flow past a rotating cylinder has been studied extensively (e.g. [1-6]), yet much continues to be unknown about the flow structure and dynamics of this geometrically simple, turbulent flow. Vortical structures in the flow and their interactions are believed to play an important role in the generation of turbulent stresses, turbulent kinetic energy, wake entrainment and growth and mean flow development. However, the direct and correct identification and observation of vertical structures and their interactions when the cylinder rotates has received little attention to date. Dol [7] and Dol et al. [8-9] have conducted a detailed experimental study, using particle image velocimeter (PIV), of the structure and behaviour of vortex shedding and vortex street for a turbulent flow over a rotating cylinder.

\footnotetext{
${ }^{\mathrm{a}}$ Corresponding author: sharulsham $@$ curtin.edu.my
}

The data obtained from PIV measurements is well suited to Proper Orthogonal Decomposition (POD) analysis. The aim is to capture most of the kinetic energy of the flow in the least number of modes possible. The snapshot POD technique is performed on instantaneous velocity fields. Proper orthogonal decomposition is performed on the velocity field data in an effort to identify and investigate time-dependent structures in the flow. The main objective of the present work is to study the breakdown of Kármán vortex street when the cylinder rotates. The interest is to examine the turbulent properties at the suppression of periodicity in vortex formation process.

\section{Experimental apparatus and technique}

The experiments were performed at the Boundary Layer Wind Tunnel Laboratory (BLWTL) of the University of Western Ontario (UWO) in a suction-type wind tunnel with a $1500 \mathrm{~mm}$ long working section and a $450 \times 450$ $\mathrm{mm}^{2}$ cross section. Hot-wire anemometry (HWA) measurements across the test section showed a uniform free-stream and a turbulence intensity of less than $1 \%$. A smooth steel circular cylinder of nominal diameter $D=$ $24 \mathrm{~mm}$ was mounted horizontally in the midplane spanning the full width of the working section. A photograph of the set-up is shown in Figure 1. The 
cylinder aspect ratio was about 18.8 so that the statistical properties of the flow were effectively two-dimensional [2]. The blockage ratio was $5.3 \%$, which is at the upper bound of the region of negligible blockage effects [1011]; no correction was made for blockage effects. A definition sketch of the flow is given in [8]. Particle image velocimetry (PIV) experiments were performed at Reynolds number of 9000 and for speed ratios, $\lambda$, between 0 and 2.7. Operating parameters for the PIV setup and measurements can be found in [7-9].

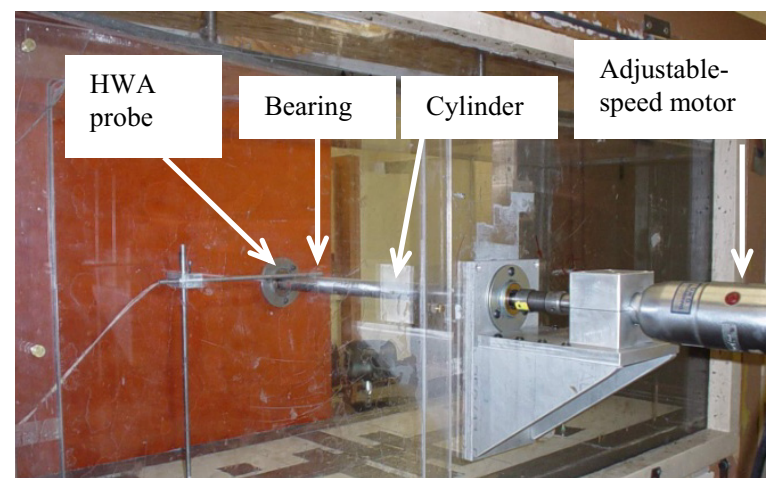

Figure 1. A photograph showing the mechanical set-up.

\subsection{POD background theory}

Let $f(x, t)$ be a random vector function on a finite spatial domain $D$ and a finite time interval $0 \leq t \leq T$. Proper orthogonal decomposition involves solving the following Fredholm-type integral equation

$$
\int_{D} R\left(x, x^{\prime}\right) \psi\left(x^{\prime}\right) d x^{\prime}=\lambda \psi(x)
$$

with $R\left(x, x^{\prime}\right)$ known as the kernel. $R\left(x, x^{\prime}\right)$ is the twopoint averaged spatial correlation function. Solution of Eq. (1) gives $\psi^{(k)}(x)$ and $\lambda_{k}$ which are the empirical eigenfunctions and eigenvalues respectively. The orthogonality condition implies that eigenfunctions of different order do not interact with each other in which case their scalar product,

$$
\psi^{(k)}, \psi^{(t)} \equiv \int_{D} \psi^{(k)}(x) \psi^{*(k)}(x) d x=\delta_{k l}
$$

is zero (* represents the complex conjugate). The original data set can be reconstructed using

$$
f(x, t) \approx \sum_{k=1}^{K} a^{(k)}(t) \psi^{(k)}(x)
$$

which is known as the proper orthogonal decomposition of $f(x, t)$.In practical situations, the data $f(x, t)$ are limited to a finite number of frames or snapshots, $M$. It can be shown that the matrix equation

$$
C \phi^{(k)}=\lambda_{k} \phi^{(k)}
$$

gives equivalent solutions to Eq. (1), [12]. Here $C$ is the spatial correlation matrix

$$
\begin{aligned}
& C_{m, n}=\frac{1}{M} \int_{D} f\left(x, t_{m}\right) f\left(x, t_{n}\right) d x, \\
& m, n=1, \ldots \ldots, M
\end{aligned}
$$

with $\phi^{(k)}$ and $\lambda_{k}$ being the eigenvectors and eigenvalues of $C$ for the $k$ th mode respectively. The empirical eigenfunctions $\psi^{(k)}(x)$ are constructed from a linear combination of the snapshots using

$$
\psi^{(k)}(x)=\sum_{n=1}^{M} \phi_{n}^{(k)} f\left(x, t_{n}\right)
$$

The problem has now been reduced to solving an $M \mathrm{x}$ $M$ matrix eigenvalue problem instead of an $N \times N$ problem (where $N$ is the number of spatial points in the domain and in most cases $N>>M$ ). This technique was first suggested by [12] and is commonly known as the method of snapshots. The direct method is computationally expensive for high-resolution data on a large spatial domain, while the snapshot method only becomes computationally expensive for large ensemble sizes (40 PIV snapshots were used in present analysis for each velocity ratio).

The energy of an instantaneous flow field variable, $f\left(x, t_{n}\right)$, represented by

$$
E_{\text {tot }}=(f, f)=\int_{D} f\left(x, t_{n}\right) f\left(x, t_{n}\right) d x
$$

The time average of the energy can thus be expressed as

$$
\left\langle E_{\text {tot }}\right\rangle=\int_{D} \overline{f(x)^{2}} d x+\left\langle\int_{D} f^{\prime}\left(x, t_{n}\right)^{2} d x\right\rangle=\left\langle E_{\text {mean }}\right\rangle+\left\langle E_{\text {fluc }}\right\rangle
$$

which can be decomposed into a mean and fluctuating component. It can be shown [13] that the energy fraction associated with the $k$ th mode, or eigenfunction $\psi^{(k)}(x)$ is 


$$
E_{k}=\frac{\lambda_{k}}{\sum_{k=1}^{M} \lambda_{k}}
$$

Using the definition of the inner product given by Eq. (2), the energy of the flow based on the velocity, $\boldsymbol{u}$ yields the following results

kinetic energy density $=\frac{1}{2} \rho(u, u)=\frac{1}{2} \rho\|u\|^{2}$

where $\rho$ is the fluid density. Hence, applying POD to $\boldsymbol{u}$ will categorize structures according to their contribution of kinetic energy to the flow.

\section{Results and discussions}

The POD modes represent the most common events occurring in the turbulent fields. Modes 1, 2, 3 and 10 from the velocity decomposition are shown in Figure 2-5 for a stationary cylinder case. In agreement with [14], the modes contribute about $22 \%, 20 \%, 13 \%, 5 \%$ and $2 \%$ respectively to the total fluctuating kinetic energy in the flow (Figure 6). It is seen that the first 2 modes have almost similar levels of energy. This result is suggestive of a less diversity of flow structure in the velocity field and results in a distinct distribution of energy between the modes and hence, an energy decomposition. A trend noticeable in the progression towards higher modes is the increase in small-scale structure within them. This can be clearly seen in the comparison between vorticity plots (curl of velocity modes) between mode 1 and mode 10, shown in Figure 2 and 5. The small scales are found to contribute only a small fraction to the fluctuating energy of the flow. However, they still appear to represent physically meaningful flow structures.

Low order representations of the instantaneous $x-y$ plane velocity fields $\omega\left(x, y, t_{n}\right)$, were reconstructed with the computed velocity modes, $\psi^{(k)}(x)$. Their corresponding vorticity distributions were compared with the distributions obtained from the PIV measurements. The reconstructions of the vorticity field using $\mathrm{k}=1,2,4$ and 10 modes are illustrated in Figure 7. The distribution obtained from the PIV for the same snapshot is also included. The figures illustrate the cumulative effect of using increasingly more modes in the data reconstruction. Good qualitative agreement of the vorticity contours is achieved with the measured distributions using approximately 4 modes in the reconstructions. As expected, the pattern of von Kármán vortices is well produced. Perrin et al. [14] found that taking $N>2$ seems to lead an overestimation of the contribution of the coherent fluctuation. A comparison between the instantaneous PIV measurements and the POD modes shows how the location of flow structures in the POD modes coincide with the location of flow structures in the PIV measurements. Large-scale structures appear to be responsible for the majority of the vortex activity. The inclusion of high-order modes (small scale structures) contribute to the fine-scale structure in the reconstructed distributions. An exaggerated vorticity distribution becomes noticeable for the 10 modes reconstruction. It seems that once all vorticity levels are recovered, the use of increasingly more modes adversely affects the reconstructions. This suggests a poor convergence of the high order modes in the decomposition which arises from noise in the PIV measurements.

Modes 1, 2, 3 and 10 from the velocity decomposition are shown in Figure 8 for $\lambda=2.0$ case. They contribute $27 \%, 6 \%, 5 \%$ and $3 \%$ respectively to the total fluctuating kinetic energy in the flow (Figure 9). It is seen that only the first mode has significant level of energy. This result is suggestive of a greater diversity of flow structure in the velocity field and results in a more even distribution of energy between the modes and hence a less efficient decomposition. The reconstructions of the vorticity field using $\mathrm{k}=1,2,4$ and 10 modes are illustrated in Figure 10. The distribution obtained from the PIV for the same snapshot is also included. Looking at the distribution of energy, where a constant decrease is observed after $N=1$, and looking at the reconstruction of the instantaneous field, it seems that taking into account the first mode would represent a good picture of the coherent picture. Dol et al. [8] found that the vortex strength is only about $10 \%$ of the initial strength at this velocity ratio. The unequal strength of the separated shear layer disrupts the vortex formation process resulting in distortion of Kármán vortex street. In other words, coherent structures are suppressed. Probably this is the reason why the vorticity structures smear over the area in larger POD modes.

The results obtained in [7-9] establish that shedding of Kármán vortices in a rotating circular cylindergenerated wake is modified by rotation of the cylinder. Alternate vortex shedding is highly visible when $\lambda<2.0$ although the strength of the separated shear layers differ due to the rotation of the cylinder. The statistics and turbulence quantities in the wakes show significant changes at $\lambda=2.0$. The results indicate that the rotation of the cylinder causes significant disruption in the structure of the flow. It is clear that flow asymmetries will weaken vortex shedding, and when the asymmetries are significant enough, total suppression of a periodic street occurs.

The average centerline velocities represent the maximum velocity deficit of the wake, $U_{\mathrm{C}}$. The velocity defect is smaller as $\lambda$ increases. $U_{\mathrm{C}} / U_{\infty}$ is about unity when $\lambda \geq 2.0$. This is due to the reduced vortex activity in the formation region [9]. This also implies that the location at which the maximum velocity defects is shifted. The mean square value, the variance (or the rms value of the fluctuation, the standard deviation) becomes smaller at higher rotational rates (especially when $\lambda \geq$ 2.0). This also means that the width of the probability density function becomes smaller (i.e. turbulent fluctuations are reduced into the vicinity of the mean value). Consequently, the turbulent intensities, Ti are also getting smaller as $\lambda$ increases due to suppression of coherent vortex structures [9]. 
mode 1

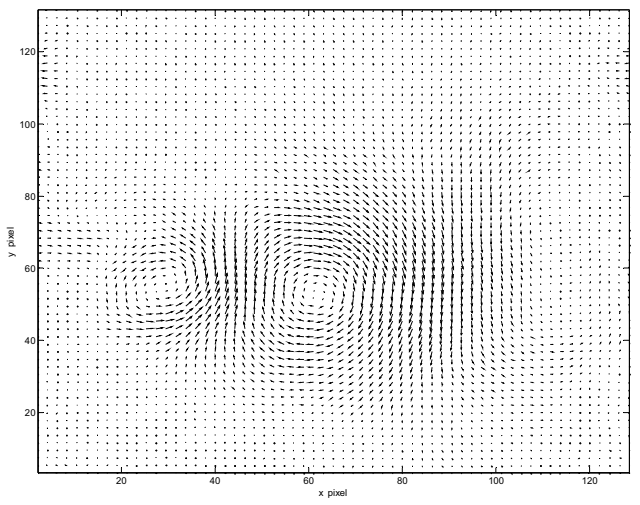

vorticity

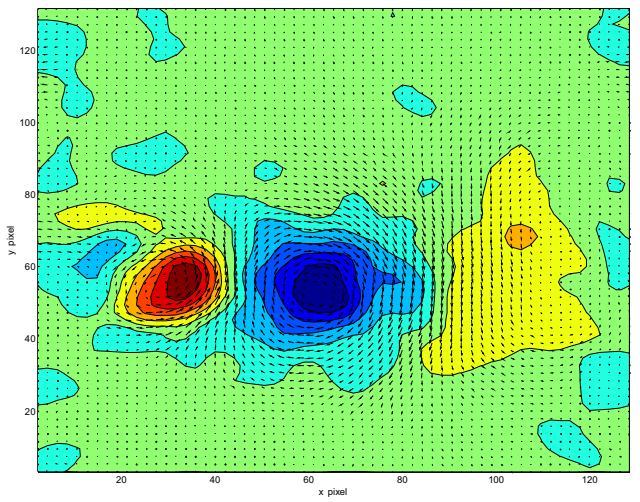

$U$ velocity

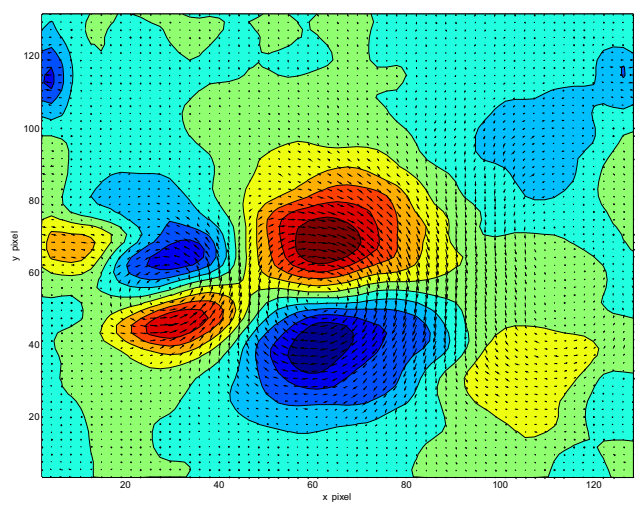

$V$ velocity

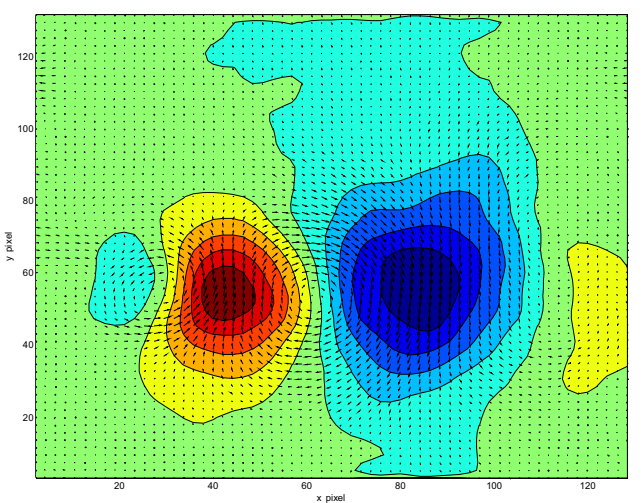

Figure 2. Mode 1 of velocity decomposition (center of the cylinder is at 0,50$)$. mode 2

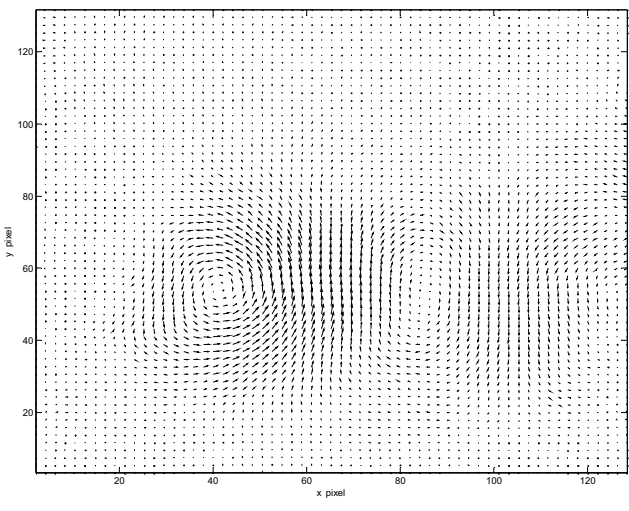

vorticity

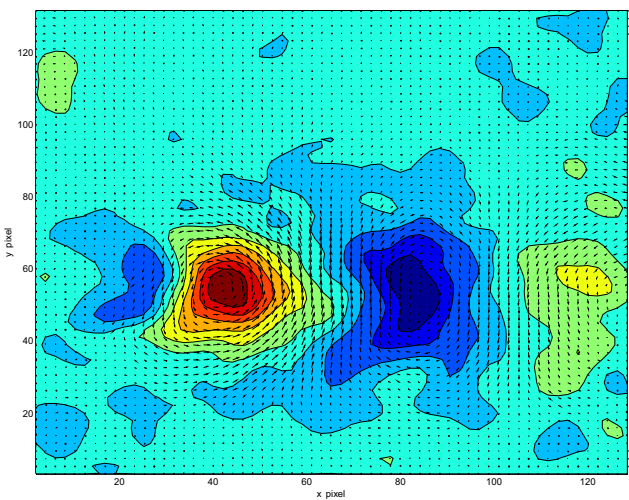

$U$ velocity

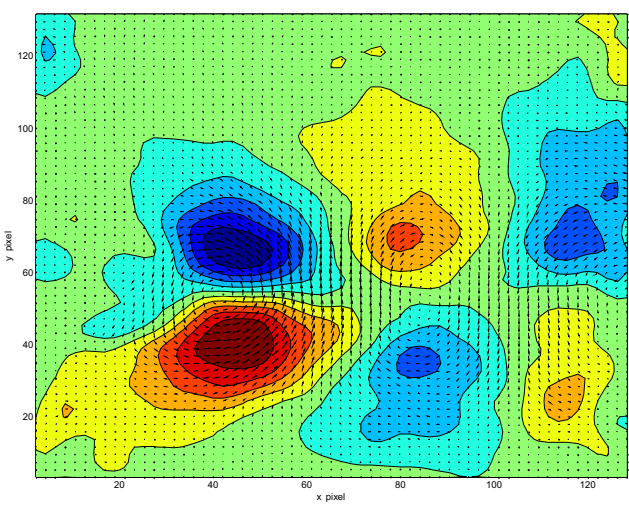

$V$ velocity

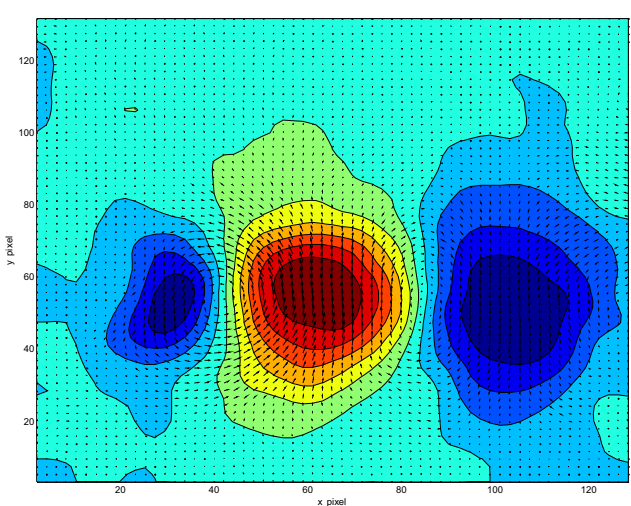

Figure 3 Mode 2 of velocity decomposition (center of the cylinder is at 0,50 ). 
mode 3

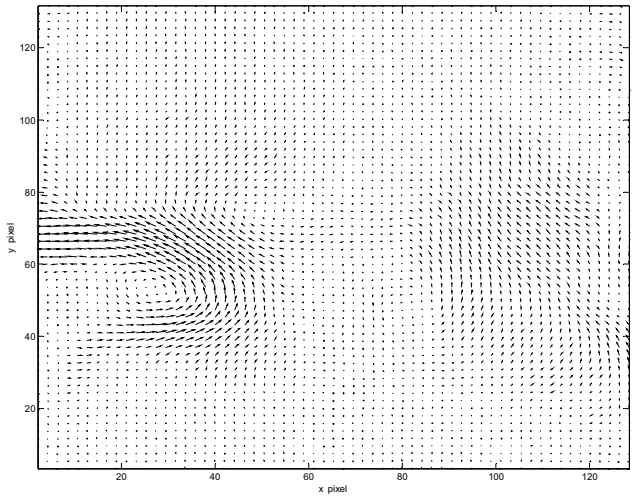

vorticity

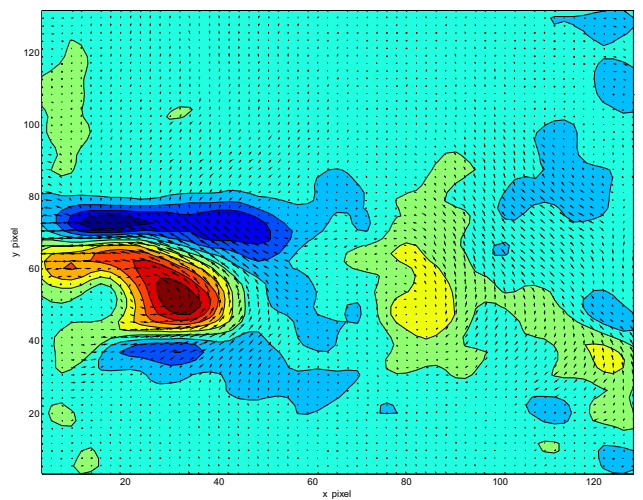

$U$ velocity

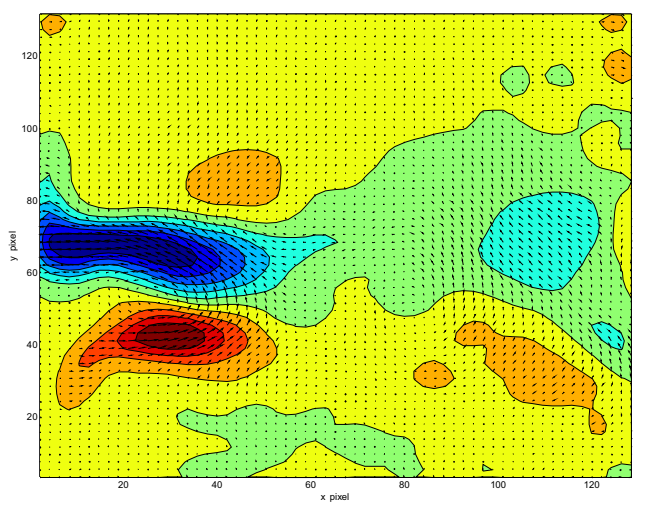

$V$ velocity

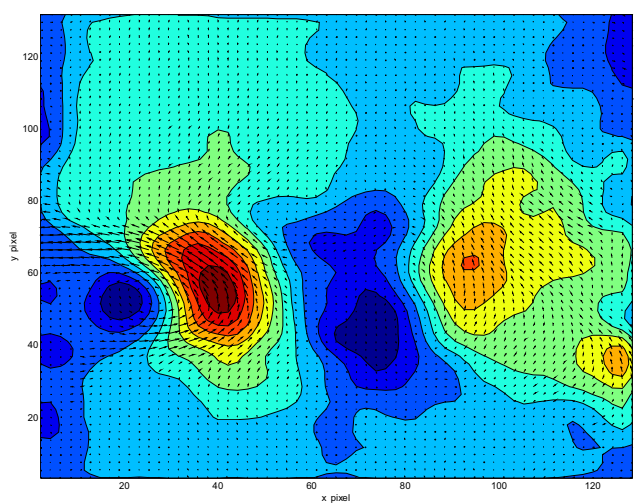

Figure 4 Mode 3 of velocity decomposition (center of the cylinder is at 0,50 ). mode 10

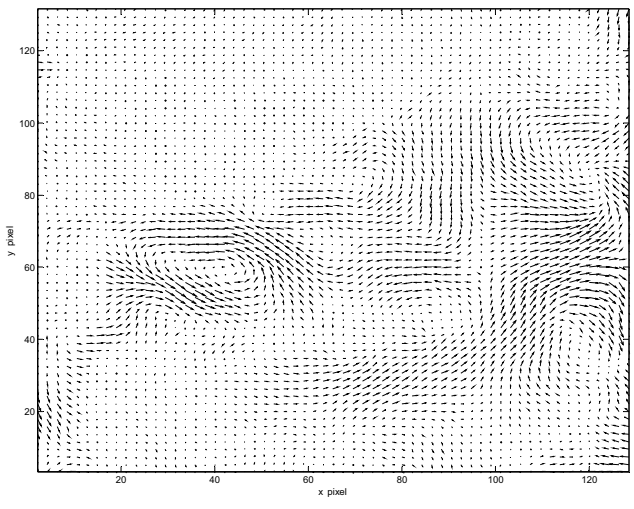

vorticity

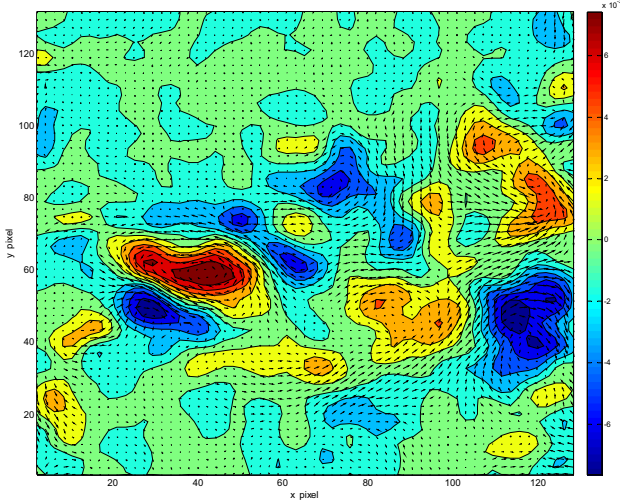

$U$ velocity

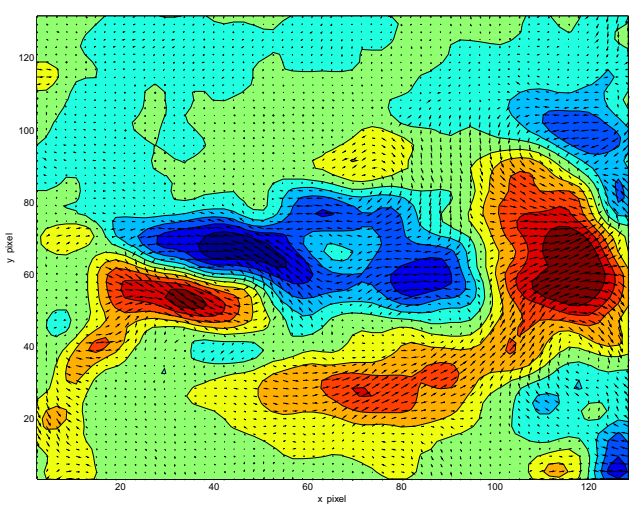

$V$ velocity

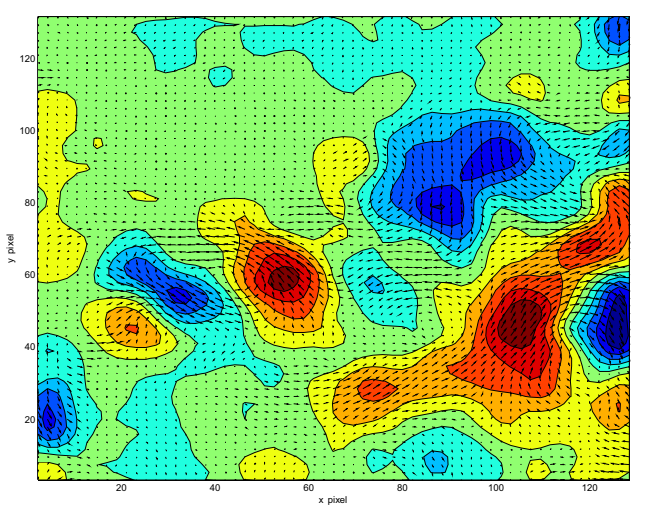

Figure 5 Mode 10 of velocity decomposition (center of the cylinder is at 0,50 ). 


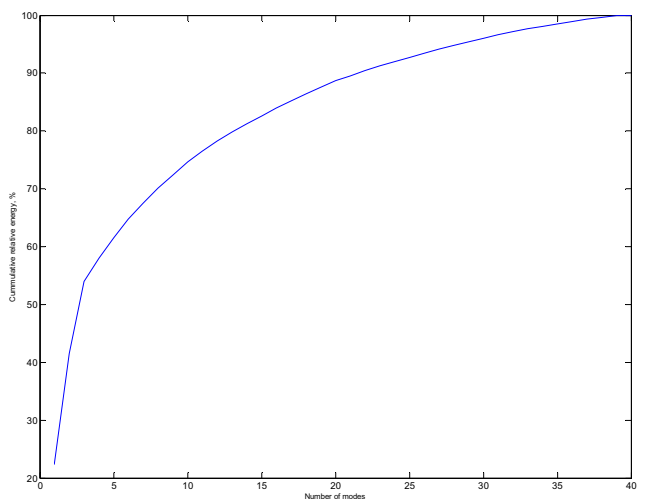

Figure 6 POD energy of the modes (in \% of the total energy of the fluctuating motion on the domain.
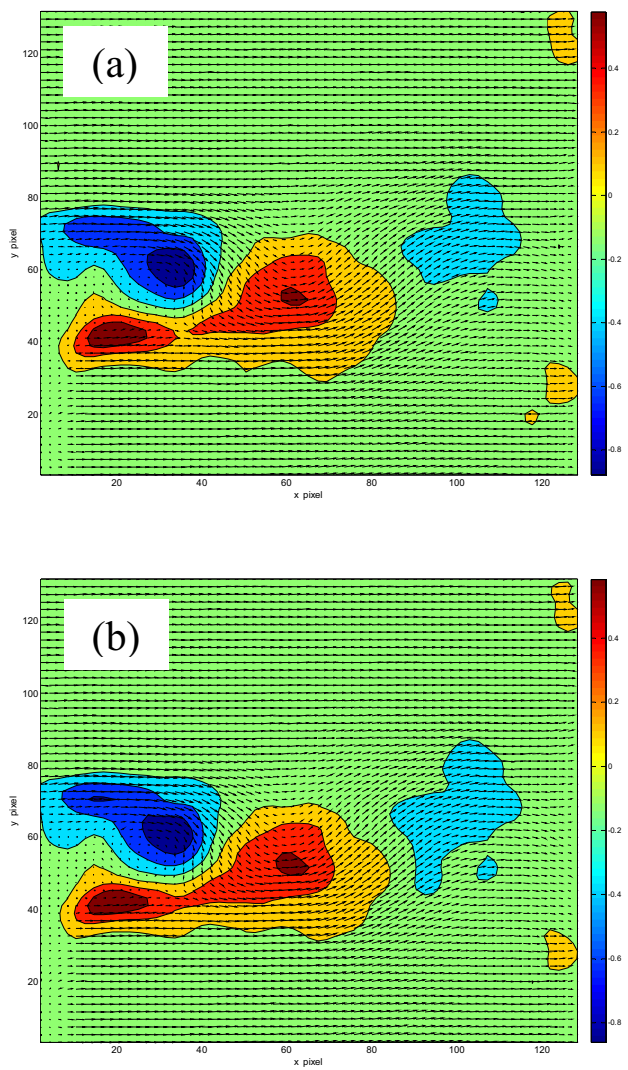
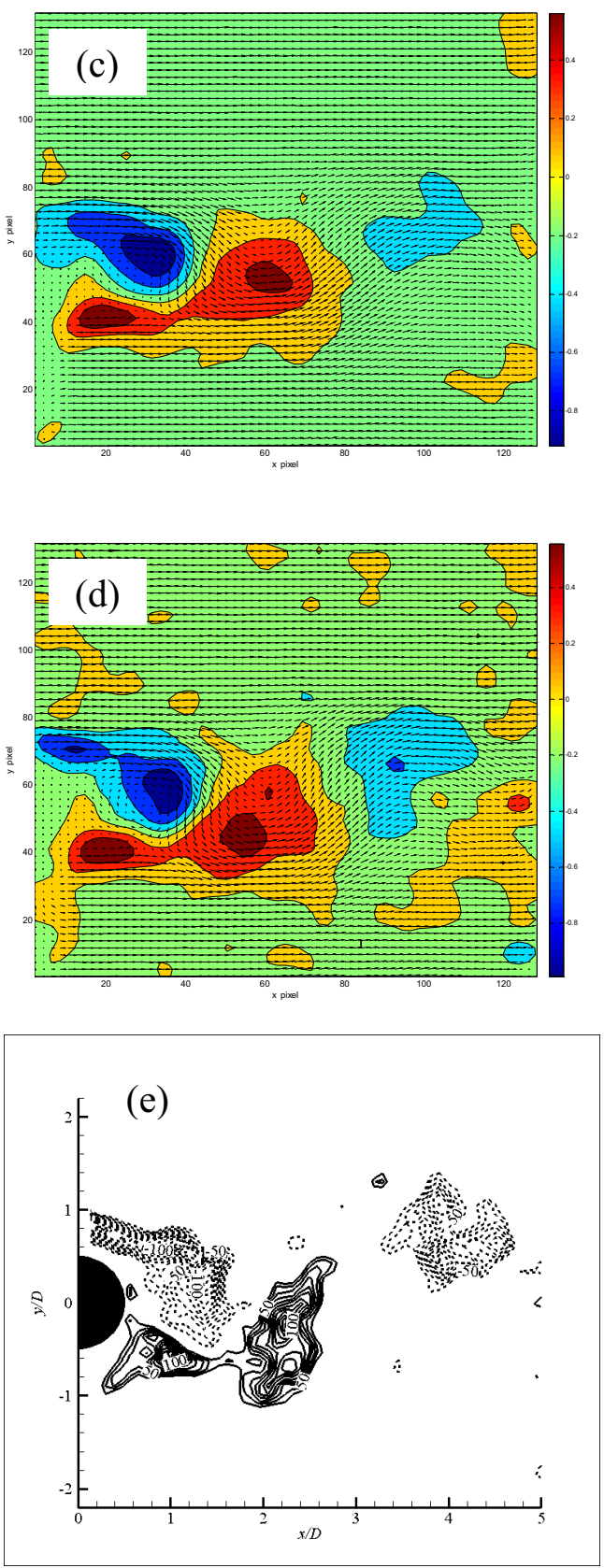

Figure 7 Vorticity distribution, obtained using (a) 1 (b) 2 (c) 4 (d) 10 modes in the reconstruction; and (e) from PIV data. 

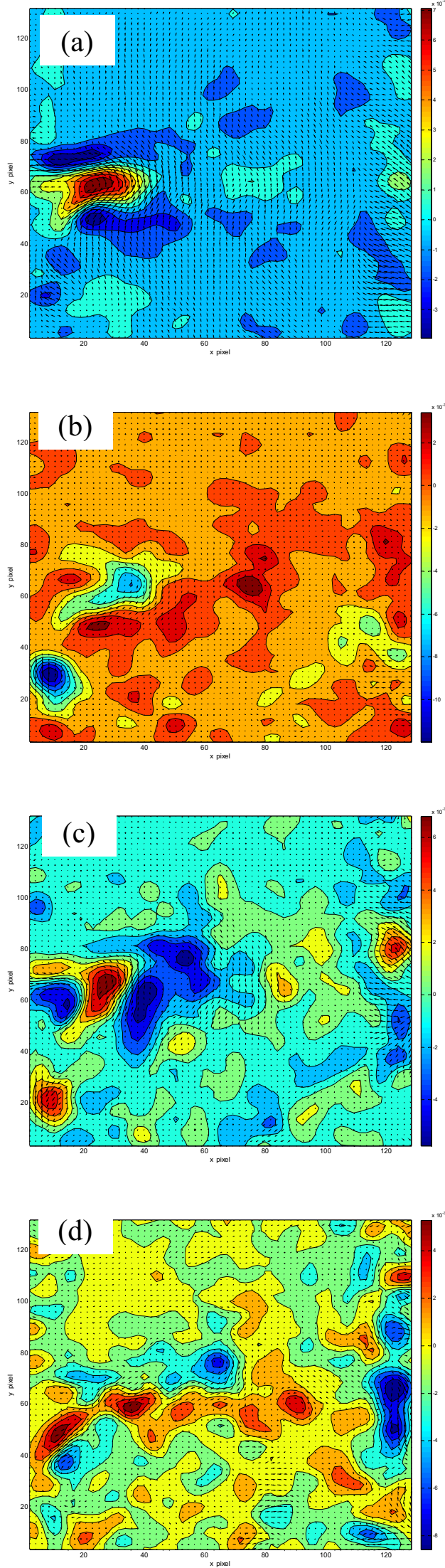

Figure 8. POD modes (a) 1 (b) 2 (c) 3 and (d) 10. Contours of vorticity are shown (center of the cylinder is at 0,50 ).

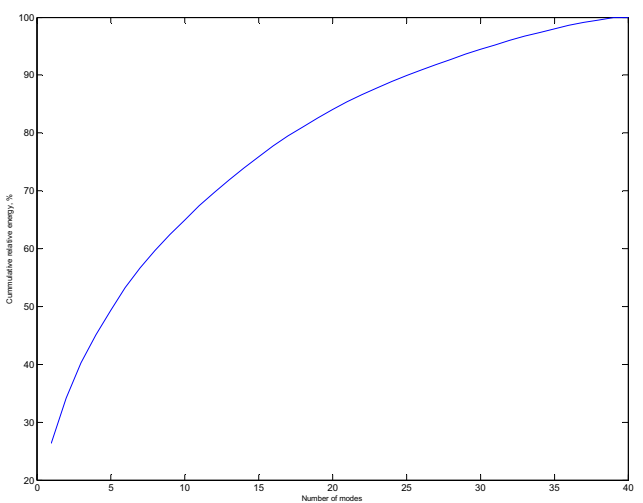

Figure 9. POD energy of the modes (in \% of the total energy of the fluctuating motion on the domain.
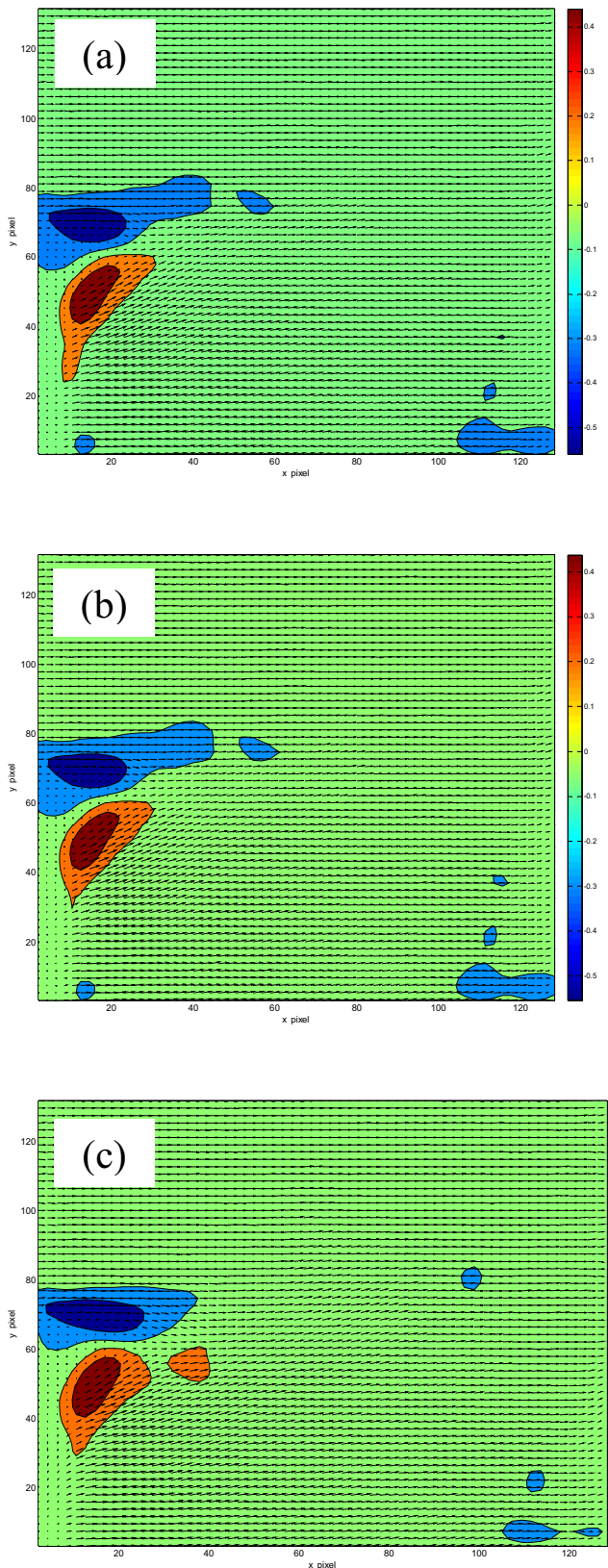

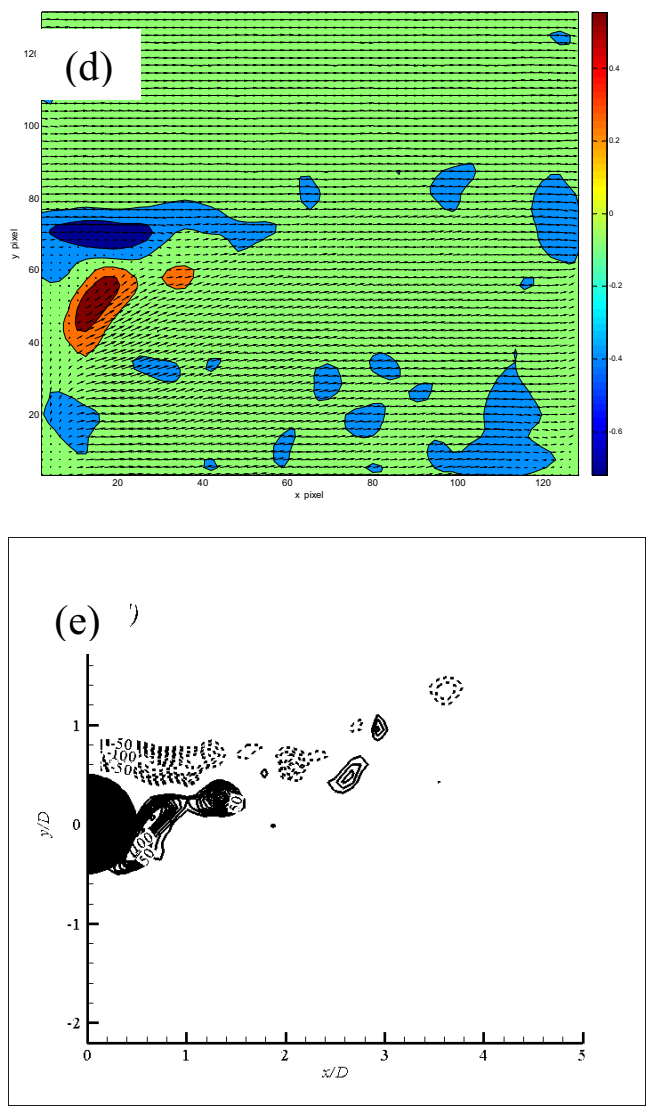

Figure 10 Vorticity distribution, obtained using (a) 1 (b) 2 (c) 4 (d) 10 modes in the reconstruction; and (e) from PIV data.

\section{Conclusion}

Turbulence studies were made in the wake of a rotating circular cylinder in a uniform free stream with the objective of describing the patterns of the vortex shedding up to suppression of the periodic vortex street at high velocity ratios. The results obtained in the present study establish that shedding of Kármán vortices in a rotating circular cylinder-generated wake is modified by rotation of the cylinder. Alternate vortex shedding is highly visible when $\lambda<2.0$ although the strength of the separated shear layers differ due to the rotation of the cylinder. The results indicate that the rotation of the cylinder causes significant disruption in the structure of the flow. Alternate vortex shedding is weak, distorted and close to being suppressed at $\lambda=2.0$. It is clear that flow asymmetries will weaken vortex shedding, and when the asymmetries are significant enough, total suppression of a periodic street occurs. Particular attention was paid to the decomposition of the flow using POD. By analyzing this decomposition with the help of PIV data, it was found that large scales contribute to the coherent motion. Vorticity structures in the modes become increasingly irregular with downstream distance, suggesting turbulent interactions are occurring at the more downstream locations, especially when the cylinder rotates.

\section{References}

1. F. Diaz, J. Gavalda, J. G. Kawall, J. F. Keffer, F. Giralt, Vortex shedding from a spinning cylinder, Physics of Fluids, 26, pp. 3454 (1983).

2. J. Massons, X. Ruiz, F. Diaz, Image processing of the near wakes of stationary and rotating cylinders, Journal of Fluid Mechanics, 204, pp. 167 (1989).

3. H. M. Badr, M. Coutanceau, S. C. R. Dennis, C. Menard, Unsteady flow past a rotating circular cylinder at Reynolds number $10^{3}$ and $10^{4}$, Journal of Fluid Mechanics, 220, pp. 459 (1990).

4. C. C. Chang, R. L. Chern, Vortex shedding from an impulsively started rotating and translating circular cylinder, Journal of Fluid Mechanics, 233, pp. 265 (1991).

5. P. T. Tokumaru, P. E. Dimotakis, Rotary oscillatory control of a cylinder wake, Journal of Fluid Mechanics, 224, pp. 77 (1991).

6. Y. T. Chew, M. Cheng, S. C. Luo, A numerical study of flow past a rotating circular cylinder using a hybrid vortex scheme, Journal of Fluid Mechanics, 299, pp. 35 (1995).

7. S. S. Dol, Weakened Vortex Shedding from a Rotating Cylinder. International Journal of Mechanical, Industrial Science and Engineering, 7(10), pp. 330-337 (2013).

8. S. S. Dol, G. A. Kopp, R. J. Martinuzzi, The suppression of periodic vortex shedding from a rotating circular cylinder. Journal of Wind Engineering and Industrial Aerodynamics, 96(6), 1164-1184 (2008).

9. S. S. Dol, U. Azimov, R. J. Martinuzzi, Statistical Description in the Turbulent Near Wake of a Rotating Circular Cylinder. World Academy of Science, Engineering and Technology, 72, pp. 575579 (2012).

10. G. S. West, C. J. Apelt, The effects of tunnel blockage and aspect ratio on the mean flow past a circular cylinder with Reynolds number between $10^{4}$ and $10^{5}$, Journal of Fluid Mechanics, 114, pp. 361 (1982).

11. A. Laneville, Turbulence and blockage effects on two dimensional rectangular cylinders, Journal of Wind Engineering and Industrial Aerodynamics, 33, pp. 11 (1990).

12. L. Sirovich, Turbulence and the dynamics of coherent structures. Part I: Coherent structures. Q Appl Mth XLV: 561-571 (1987).

13. P. Holmes, J. Lumley G. Berkooz, Turbulence, coherent structures, dynamical systems and symmetry. Cambridge University Press, Cambridge (1996).

14. R. Perrin, M. Braza, E. Cid, S. Cazin, A. Barthet, A. Sevrain, C. Mockett, F. Thiele, Obtaining phase averaged turbulence properties in the near wake of a circular cylinder at high Reynolds number using POD. Exp Fluids 43:341-355 (2007). 